\title{
The choice of fluid and thermal grid of the organic Rankine cycle in the conditions of its application at the oil refineries
}

\author{
M.A. Peretyatko \\ Ph.D. student, Saint-Petersburg Mining University, Saint-Petersburg, Russia \\ P.V. Yakovlev \\ Professor, Saint-Petersburg Mining University, Saint-Petersburg, Russia
}

V.A. Lebedev

Professor, Saint-Petersburg Mining University, Saint-Petersburg, Russia

\author{
A.S. Deev \\ Postgraduate student, Saint-Petersburg Mining University, Saint-Petersburg, Russia
}

\begin{abstract}
The article discusses the problem of choosing the working fluid for the Rankine organic cycle. This is done by analyzing the parameters of the ORC unit on these Freon's. It is proposed to use low-grade heat of technological units for oil refining as a heat source for the organic Rankine cycle unit. Currently, a significant part of the available lowgrade heat from oil refineries is lost into the atmosphere. The installation of the organic Rankine cycle allows the use of this heat to generate an expensive energy resource - electricity. This is the advantage of this installation over other methods of utilizing low-grade heat. At the moment, there is no clear methodology for choosing a working fluid for the organic Rankine cycle, that used at an oil refinery. For comparison, the following freons were selected: $245 \mathrm{fa}$, r152a and r404a. To solve this problem, a model that calculates such indicators as thermal efficiency, coolant flow rate and electric power of the installation, for the simplest scheme of the organic Rankine cycle was built. The article also provides an analysis of the results obtained by modeling the comparison of the considered working bodies. To assess the feasibility of using the unit with selected coolant, the economic effect of its implementation was evaluated.
\end{abstract}

\section{INTRODUCTION}

In the modern world, more and more attention is paid to the problem of improving energy efficiency. One of the tasks of increasing energy efficiency is the development of methods for recycling of low-grade heat. Low-potential heat sources include flows of various substances and gases with a temperature of not more than $300^{\circ} \mathrm{C}$. Such sources are very common in the oil, metallurgical chemical and other industries.

Consider the refining industry in more detail. The technological process of oil refining is not perfect in terms of the full use of thermal energy. Thermal energy supplied to oil at refineries is used only by $30 \%$. About $35 \%$ of it discharged into the atmosphere using circulation water system or air-cooling units, about $10-15 \%$ released into the atmosphere from surfaces of equipment and about 15\% released into the atmosphere with flue gases (Burdygina, 2003). According to estimates, at refineries with a capacity of 5-6 million tons per year of crude oil, at least $15 \mathrm{MW}$ of low potential heat with a temperature of at least $150^{\circ} \mathrm{C}$ are discharged into the atmosphere.

One of the modern ways of recycling this low-grade heat is organic Rankine cycle (ORC). $\mathrm{ORC}$ is used to generate electricity. The schematic diagram of the OCR unit is shown in Figure 1. 


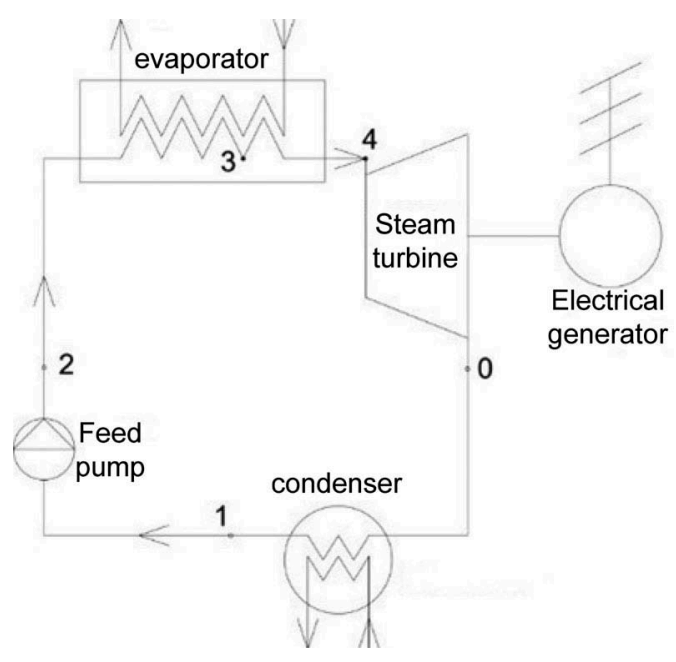

Figure 1. Schematic diagram of the OCR unit.

OCR works on the same principle as the classical Rankine cycle with water as a fluid. The main difference between organic fluid and water is a low boiling point, which allows steam to be generated at low temperatures of heat sources.

For the normal operation of a steam turbine, it is necessary, to have a large pressure difference, which is different for various fluids. In this regard, depending on the temperature conditions of the thermal scheme of the organic Rankine cycle, it is necessary to determine the compound that will be used as a working fluid. When choosing different compounds, you must follow rules:

- Work, done by steam in the turbine, should be as much as possible;

- Thermal conductivity of the fluid should be as large as possible for more efficient heat transfer in the evaporator and condenser;

- Fluid must be stable at high temperatures;

- Fluid should be environmentally friendly and safe.

Based on these criteria, for further consideration, the following freons were selected: $245 \mathrm{fa}$, r152a, r404a. The parameters of the considered working fluid are presented in Table 1.

These freons have already been used as a coolant for ORC, but under other conditions. It is advisable to consider them for specific conditions of use at a refinery.

One of the main indicators of the effectiveness of the Rankine cycle is the thermal efficiency of the cycle. In this case, the thermal efficiency of the cycle will depend on the selected compound. Based on this fact, the choice of the working fluid will be made according to the highest thermal efficiency of the cycle. Further in the work, an unit working on the selected fluid will be considered.

Consider freon $\mathrm{r} 245 \mathrm{fa}$ as a working fluid for the proposed heat recovery unit at oil refineries. The p-h diagram of the ORC with r $245 \mathrm{fa}$ is shown in Figure 2.

Table 1. Parameters of the considered working fluid.

\begin{tabular}{llll}
\hline & \multicolumn{2}{l}{ Value } & \\
\cline { 2 - 4 } Parameter & r245fa & r152a & r404a \\
\hline Molar mass, g/mol & 134,1 & 66,1 & 97,6 \\
Boiling point, ${ }^{\circ} \mathrm{C}$ & 15,3 & -24 & $-46,3$ \\
Specific thermal capacity in a liquid state at normal pressure, $\mathrm{kJ} / \mathrm{kg} \mathrm{K}$ & 1,36 & 1,8 & 1,62 \\
Ozone depletion potential & 0 & 0 & 0 \\
Global warming potential & 1030 & 140 & 3750 \\
\hline
\end{tabular}




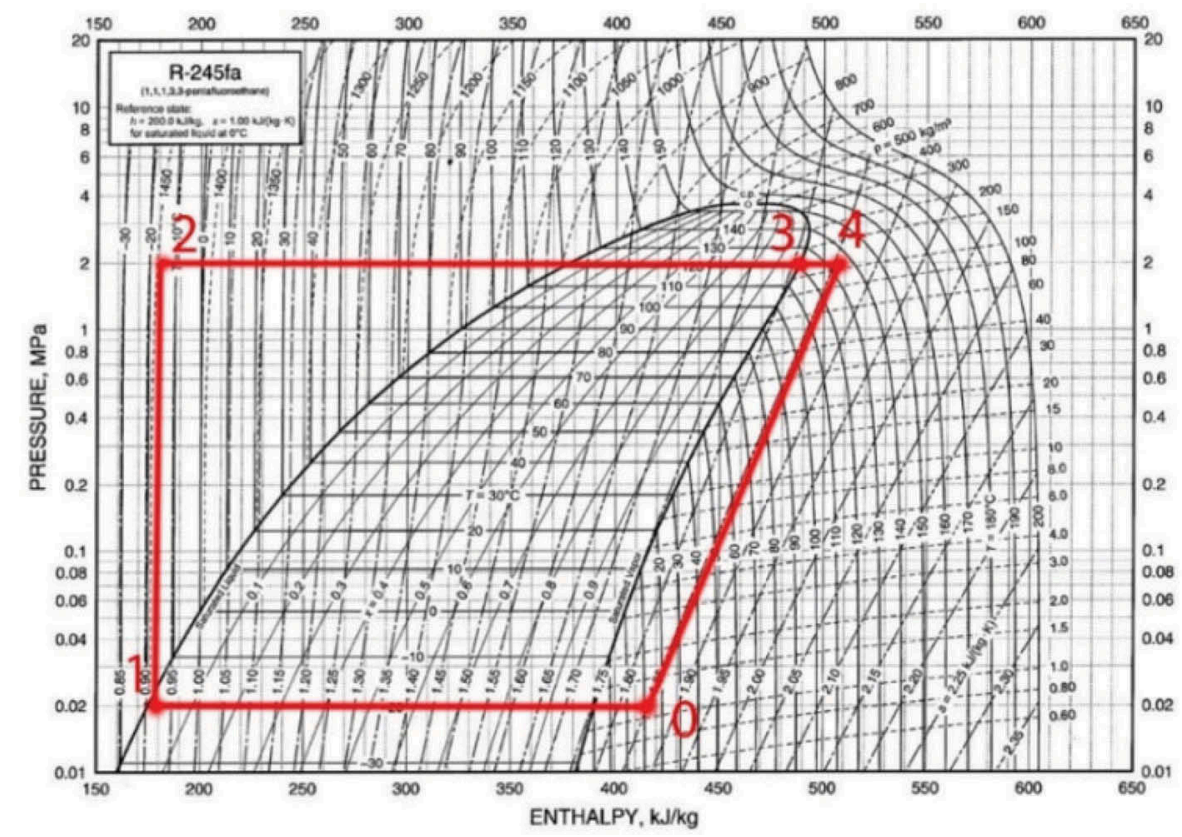

Figure 2. P-h diagram of the ORC with r245fa.

The following algorithm was used to determine the installation efficiency:

- The value of the temperature of the quenching compound in the condenser and power of the low-potential heat source and its temperature are set. The temperature of the lowgrade heat source determines the maximum attainable temperature of the organic fluid;

- The enthalpies of the fluid at the characteristic points of the cycle are determined. The characteristic points of the cycle on r245fa are presented in Table 2;

- Further, the thermal efficiency of the unit, the work done by steam in the turbine and its adiabatic power are determined from the values of enthalpies in the evaporator and condenser;

- Then, the power of the feed pump is determined. Power of the ORC unit, taking into account the operation of the feed pump, the energy loss in the generator and the internal efficiency of the turbine is determined.

Then, in a similar way, calculation was performed for the remaining freons. To facilitate the calculations, a model of the OCR unit was built in Microsoft Excel. This model automatically calculates all parameters of the cycle after entering the initial data. ${ }^{\circ} \mathrm{C}$

For comparison, several freons as a working fluid in the ORC, the temperature range of the quenching compound is assumed to be the same (from $-20^{\circ} \mathrm{C}$ to $20^{\circ} \mathrm{C}$ ), as well as the values of the internal efficiency of the turbine $(86 \%)$ and the efficiency of the generator $(95 \%)$. Then we

Table 2. Characteristic points of the cycle on r245fa.

\begin{tabular}{lll}
\hline Point & Enthalpy, $\mathrm{KJ} / \mathrm{kg}$ & Note \\
\hline 0 & 410 & End of expansion in a turbine \\
1 & 175 & At the exit of the condenser \\
2 & 185 & At the entrance to the evaporator \\
3 & 480 & At the exit of evaporator \\
4 & 505 & At the exit of the superheater \\
\hline
\end{tabular}


compare technical performance of the unit with different fluids. The most suitable compound is the one at which the power of the unit is greatest.

One of the problems of ORC is low efficiency. Thermal efficiency of these installations usually does not exceed 28\% (Casati, 2014). One of the methods for increasing efficiency is the improvement of the cycle arrangement. However, this method leads to a complication of the design of the unit and an increase in its cost. For this reason, we built computer model that allows you select the most optimal cycle diagram of the ORC unit. The selection is made according to the following principle:

- The technical parameters of three cycle diagrams are calculated (simple circuit, circuit with economizer, circuit with intermediate steam overheating and economizer);

- Economical indicators of each circuit are determined;

- The optimal cycle diagram is selected based on the economic performance.

The calculation of the technical indicators of the unit without intermediate overheating of the steam and with economizer was carried out similarly to the algorithm for choosing the working fluid. The difference is that in this calculation the power of the feed pump is not taken into account.

Consider the OCR unit with an economizer. The thermal circuit of this unit is shown on Figure 3.

This scheme is used to increase the efficiency of the cycle. Economizer is installed between the turbine and the condenser and it heat the condensate after the feed pump in front of the evaporator by cooling the steam leaving the turbine to a condensation temperature $20^{\circ} \mathrm{C}$.

The calculation of the technical indicators of the unit with an economizer was carried out similarly to the above algorithm; however, the calculation is complicated due to the need to make the heat balance of the economizer. From the heat balance equation, the enthalpy of the heated refrigerant at the outlet of the economizer is determined.

Next, a thermal circuit is calculated with an intermediate superheat of the steam and an economizer (Figure 4).

The calculation of the technical indicators of the unit operating according to this thermal scheme was carried out similarly to the calculation of the scheme with the economizer; however, the calculation is complicated by adding two characteristic points of the cycle: after high-pressure section and before low pressure section. It is necessary to set the value of the pressure of steam after the high-pressure section ( $0.6 \mathrm{MPa}$ in this case), as well as to compile the heat balance of the intermediate superheater of the steam. The initial enthalpy of steam in the low pressure section is calculated from the heat balance equation.

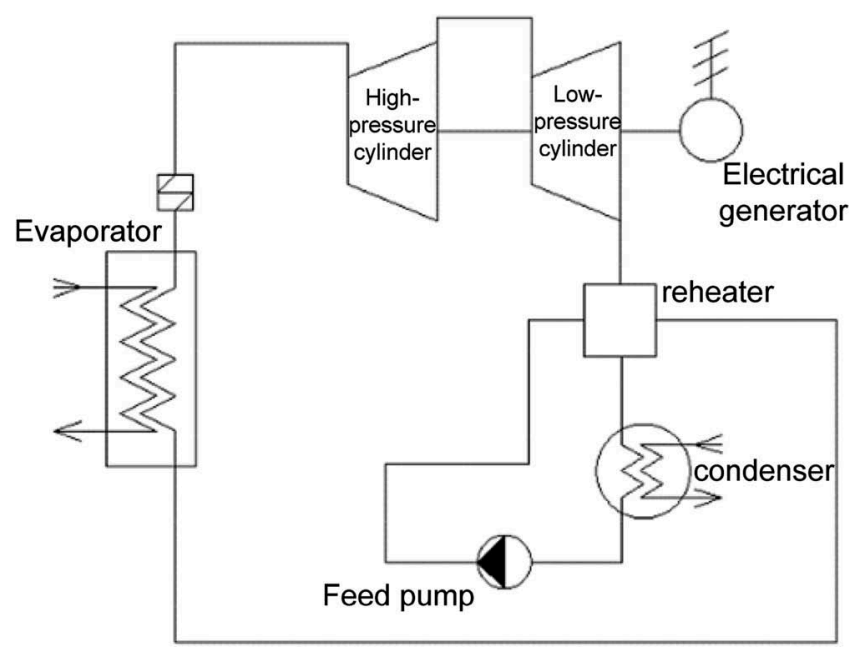

Figure 3. Thermal circuit of OCR unit with an economizer. 


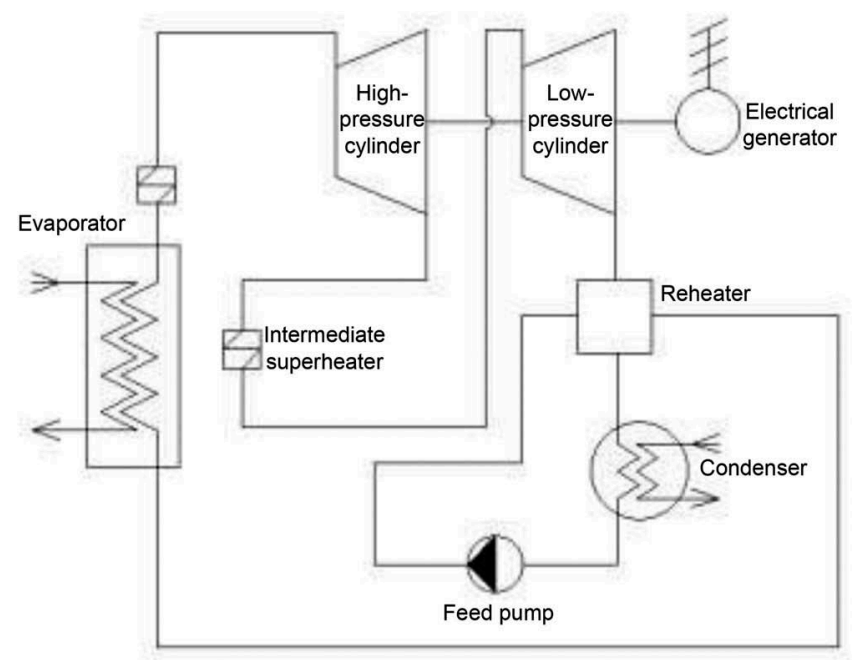

Figure 4. Thermal circuit of OCR unit with an intermediate superheat of the steam and an economizer.

Next, the economic indicators of the installation are determined. The calculation formulas for determining the indicators of economic efficiency are presented in Table 3.

The main indicators of economic efficiency is discounted payback period, discounted Profitability Index, internal rate of return and net present value.

Table 3. Calculation formulas for determining the indicators of economic efficiency.

\begin{tabular}{ll}
\hline Calculation period (T), years & 15 \\
\hline Depreciation (A), thousand rubles a year & $A=C / T^{1}$ \\
Cash Flows (cost of saved electricity), $\mathrm{CF}_{\mathrm{t}}$, thou- & $C F_{t}=c_{k h} T_{\text {work }} N^{2}$ \\
sand rubles a year & \\
Operating expenses (O), thousand rubles a year & $O=0,05 C$ \\
Average employee wages (w), thousand rubles & 600 \\
a year & \\
Number of employers, n & 8 \\
Wages expenses (W), thousand rubles a year & $W=w n$ \\
Social expenses (S), thousand rubles a year & $S=0,3 W$ \\
Cost of equipment (PE), thousand rubles & $P E=C-A t^{3}$ \\
Property tax (WT), thousand rubles a year & $W T=0,022 P E$ \\
Balance (B), thousand rubles a year & $B=C F-C-C I W-D S W-A-O-W-S-W T$ \\
Profit tax (PT), thousand rubles a year & $P T=0,2 B$ \\
Net cash flow (NCF $),$ thousand rubles a year & $N C F_{t}=B-P T$ \\
Discount rate (i) & $i=i_{0}+i_{r}{ }^{4}$ \\
Net present value (NPV), thousand rubles & $N P V=\sum_{t=0}{ }^{N} \frac{N C F_{t}}{(1+i)^{t}}$ \\
Internal rate of return (IRR) & IRR=i, wherein $N P V=0$ \\
Discounted profitability Index (DPI) & $D P I=N P V+C$ \\
Discounted payback period (DPP) & DPP=t, wherein $N P V=0$ \\
\hline
\end{tabular}

1 Where C - capital expenses

2 Where $c_{k h}$-cost kilowatt hour, $T_{\text {work }}$-Time of work, $N$-power

3 Where t- period number

4 Where: $i_{0}=0,07$ - risk-free rate, $i_{r}=0,02$-risk rate 


\section{RESULTS}

The dependences of thermal efficiency and electric power on the temperature of the cooling medium, which were obtained as a result of calculations (Figure 5).

The obtained dependences of electric power on the temperature of the cooling medium are presented in Figure 6.

As we can see from the Figure 5 and 6, efficiency and electric power are highly dependent on temperature of the cooling medium: power decreases with average speed $40 \mathrm{~kW} /{ }^{\circ} \mathrm{C}$; efficiency decreases with average rate of $0.27 \% /{ }^{\circ} \mathrm{C}$. Unit with freon $\mathrm{r} 245 \mathrm{fa}$ as a fluid, have the greatest power and efficiency rate.

Thermal efficiency and electric power for each of the three considered thermal circuits were determined. These indicators are presented in Figure 7.

As expected, a thermal circuit with an economizer and intermediate steam superheating has the highest energy efficiency. However, it should be noted that the easiest way to increase the efficiency of the ORC unit is to add an economizer. This method increases the thermal efficiency by $2.2 \%$ and the unit power by $300 \mathrm{~kW}$.

Indicators of economic efficiency of the project are presented in table 4. NPV for the periods of operation is presented in Figure 8.

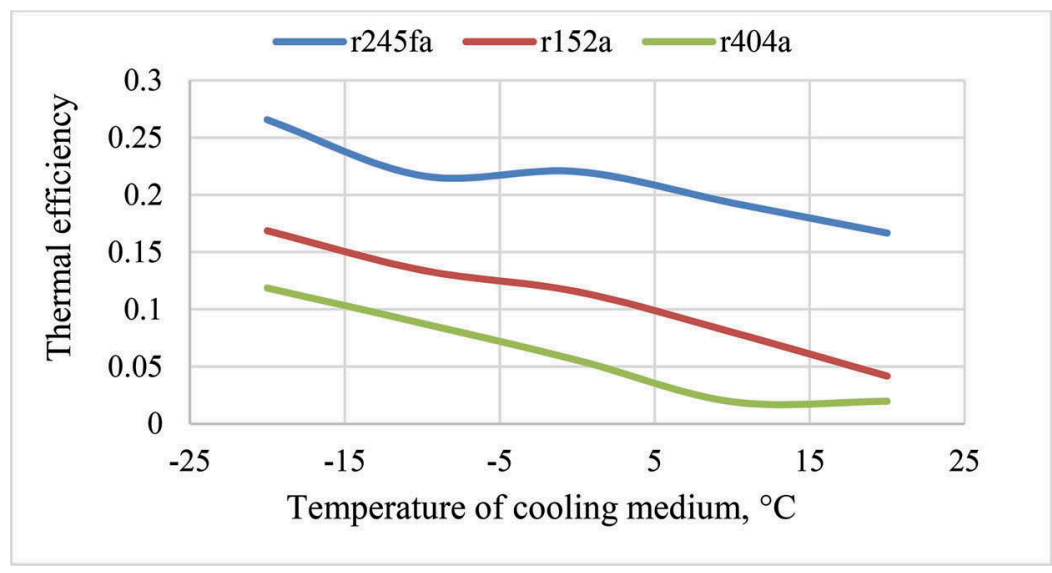

Figure 5. The dependences of thermal efficiency and electric power on the temperature of the cooling medium.

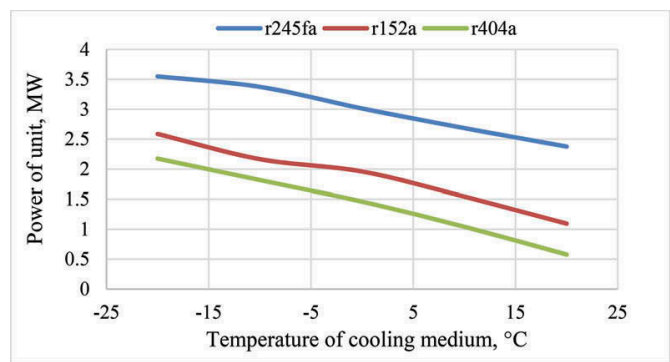

Figure 6. Dependences of electric power on the temperature of the cooling medium. 


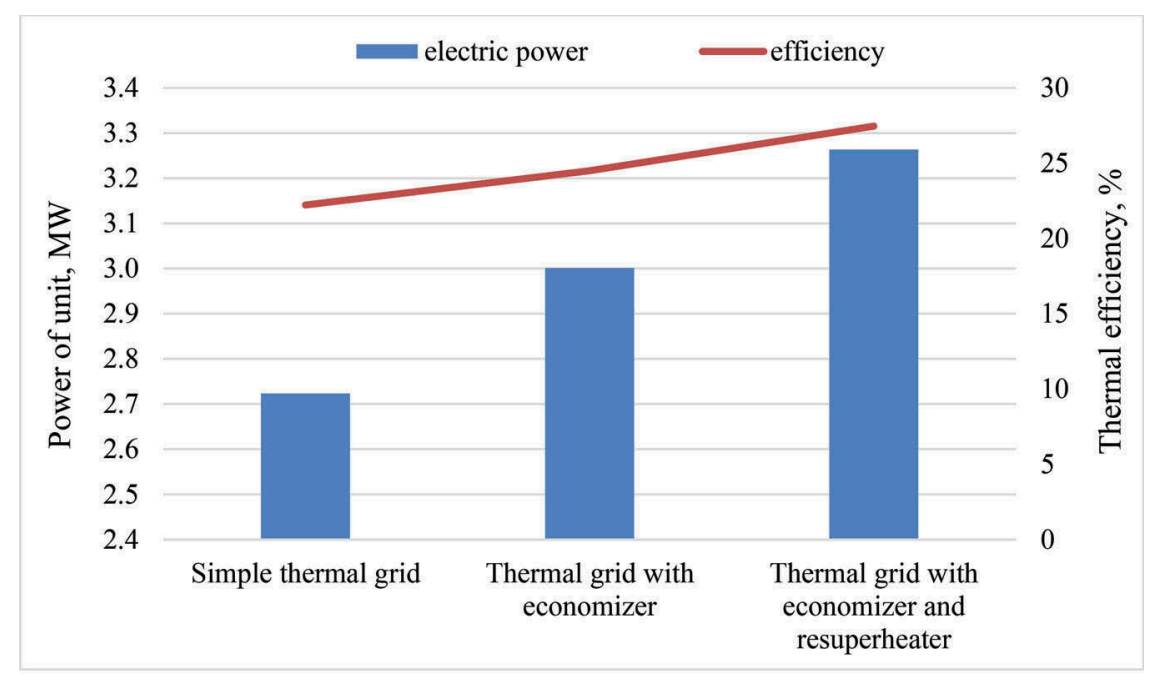

Figure 7. Indicators of energy efficiency each of the three considered thermal circuits.

Table 4. Indicators of economic efficiency.

\begin{tabular}{llll}
\hline Variant & $\begin{array}{l}\text { Without economizer, without } \\
\text { intermediate superheater }\end{array}$ & $\begin{array}{l}\text { With economizer, without } \\
\text { intermediate superheater }\end{array}$ & $\begin{array}{l}\text { With economizer, with } \\
\text { intermediate superheater }\end{array}$ \\
\hline NPV, thou- & 124887 & 158630 & 138413 \\
sand rubles & $24,94 \%$ & $24,29 \%$ & $23,59 \%$ \\
IRR & 1,55 & 1,55 & 1,49 \\
DPI & 6,69 & 7,15 \\
DPP, year & 6,69 & & \\
\hline
\end{tabular}

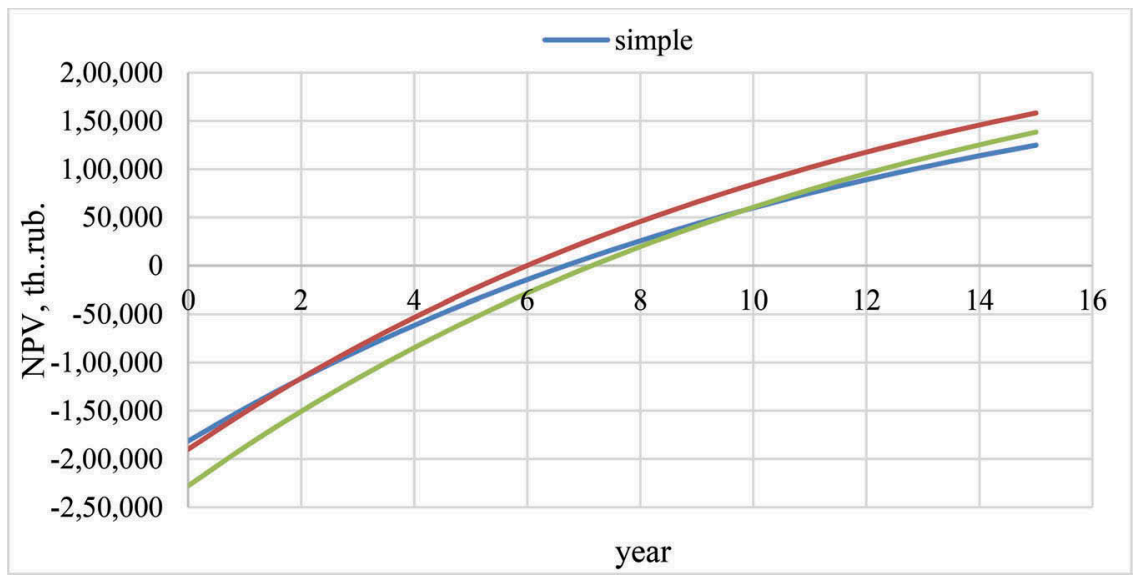

Figure 8. Indicators of economic efficiency. 


\section{DISCUSSION OF THE RESULTS}

From Figures 5 and 6, we can see that the cycle on $\mathrm{r} 245 \mathrm{fa}$ freon has the highest values of thermal efficiency and electric power (in the temperature range of the cooling medium from $-20^{\circ}$ $\mathrm{C}$ to $20^{\circ} \mathrm{C}$ ), thus, we find that it turned out to be the most suitable fluid for given initial conditions.

Figure 7 shows that the thermal circuit with an intermediate superheater and economizer is expected to be better than the other considered options for thermal circuits in terms of the full use of the heat of the source. The thermal efficiency of this option is 3.0\% higher than that of a thermal circuit with an economizer and 5.2\% higher than that of thermal circuit without auxiliary equipment. It should also be noted that installation of the economizer increases the thermal efficiency of the unit by $2.3 \%$ with the relatively low cost of this element, the simplicity of its installation and operation.

Now we are comparing the economic indicators of the unit options under consideration. As it can be seen from Table 4 and Figure 8, despite the greatest energy efficiency, a thermal circuit with an intermediate superheater and economizer is the least profitable in terms of economic indicators. This thermal scheme has the worst values of DPI, DPP and IRR. The reason for the economic inexpediency of using this thermal scheme is the high cost of the system components (a significant increase capital expenses, the cost of installation and operation of a turbine unit with intermediate superheater).

Interesting results are obtained by comparing the economic indicators of units without an economizer and superheater with the unit with an economizer and without superheater. As can be seen from Table 4 and Figure 8, despite the lower efficiency of the first variant (thermal efficiency is lower by $2.3 \%$ ), the economic performance indicators for this variant are not much worse than the option with the economizer installed. However, if we consider the dependence of NPV on the lifetime, you can also notice that with an increase in the operating life, the most energy-efficient variant begins to approach two other variants and, starting from the 9 th year of operation, surpasses the least energy-efficient variant.

\section{CONCLUSION}

In this paper, we considered the problem of choosing a working fluid and the thermal circuit of the OCR for refinery industry. For this, an algorithm was developed that allows you to choose the most effective variant from the point of view of its technical and economic parameters.

The described method can also be used to select a working fluid for the ORC under other initial conditions. It is universal for all ranges of powers and temperatures of low-grade heat sources.

With the help of this algorithm, the most acceptable variant of the Thermal circuit of OCR was chosen for the given initial conditions. This is a variant of the thermal circuit of the OCR with an economizer, despite its lower energy efficiency compared to the variant of the thermal circuit with an economizer and intermediate steam overheating. Also, based on the analysis of the results obtained, an assumption was made: with an increase in the power of the lowpotential heat source and the working lifespan, the most energy-efficient option will be more and more economically feasible. This statement is due to the following: the NPV of this variant will increase faster than that of other options with an increase in the working lifespan, and also an increase in the unit capacity of the unit will lead to a decrease in the unit cost of equipment.

Economic indicators from the implementation of the proposed technology prove the feasibility of its use. This technology allows the most efficient use of the energy resources of the plants, which ultimately leads to a reduction in the cost of finished products. 


\section{REFERENCES}

Burdygina, E.V. 2003. Improving the energy efficiency of heat engineering equipment for crude oil distillation. Authors abstract. Ufa: Ufa State Petroleum Technological University.

Chen, H. \& Goswami, D.Y. \& Stefanakos, E.K. 2010. A rewiew of thermodynamic cycles and working fluids for the conversion of low-grade heat. Renewable and Sustainble Energy Reviews Vol. 14: pp. 3059-3067.

Emiliano, I.M. Casati. 2014. New concepts for Organic Rankine cycle power systems. Milan: Politecnico di Milano

Fitsak, V.V. \& Boguslavskiy, E.I. 2017. Technology and economics of near-surface geothermal resources exploitation. Journal of Mining Institute Vol. 224: pp. 189-198.

Kang, S. 2012. Design and experimental study of ORC and radial turbine using R245fa working fluid. Energy №41: pp. 514-524.

Kirsanova, N.Y. \& Lenkovets, O.M. \& Nikulina, A.Y. 2018. Renewable energy sources (RES) as a factor determining the social and economic development of the arctic zone of the Russian Federation. International Multidisciplinary Scientific GeoConference Surveying Geology and Mining Ecology Management, SGEM Vol. 18: pp. 679-686.

Makhovikov, A.B. \& Katuntsov, E.V. \& Kosarev, O.V. \& Tsvetkov, P.S. 2018. Digital transformation in oil and gas extraction. Innovation-Based Development of the Mineral Resources Sector: Challenges and Prospects - 11th conference of the Russian-German Raw Materials Vol. 1: pp. 531-538.

Russian Federation Federal Law No. 261-FZ of November 23, 2009. On Energy Saving and Increase of Energy Efficiency and Introduction of Changes into Separate Legislative Acts of the Russian Federation

Smirnova, N.N. \& Izotov, E.A. 2017. Methodology for estimating heat losses due to heat effects on a heterogeneous oil reservoir. Journal of Industrial Pollution Control Vol. 33: pp. 950-958.

Smirnova, N.V. \& Rudenko, G.V. 2017. Tendencies, problems and prospects of innovative technologies implementation by Russian oil companies. Journal of Industrial Pollution Control Vol. 33: pp. 937-943.

Tartiere, T. \& Astolfi, M.A. 2017. World overview of the organic Rankine cycle market. Energy Procedia Vol. 129: pp. 2-9. 